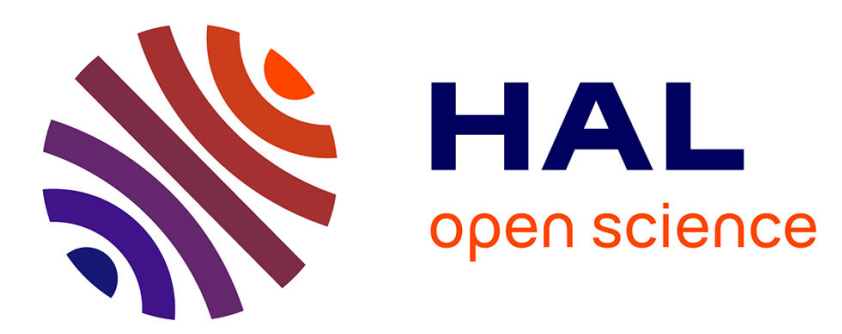

\title{
Real-time Accelerometer Coupled Self-Mixing Laser Displacement Sensor for Embedded Applications
} Usman Zabit, Olivier Bernal, Alexandro Chamorro-Coloma, Thierry Bosch

\section{To cite this version:}

Usman Zabit, Olivier Bernal, Alexandro Chamorro-Coloma, Thierry Bosch. Real-time Accelerometer Coupled Self-Mixing Laser Displacement Sensor for Embedded Applications. IEEE Sensors Conference 2012 Taipei, Oct 2012, Taipei, Taiwan. pp.IEEE Sensors Conference 2012, 10.1109/ICSENS.2012.6411493 . hal-00801203

\section{HAL Id: hal-00801203 https://hal.science/hal-00801203}

Submitted on 15 Mar 2013

HAL is a multi-disciplinary open access archive for the deposit and dissemination of scientific research documents, whether they are published or not. The documents may come from teaching and research institutions in France or abroad, or from public or private research centers.
L'archive ouverte pluridisciplinaire HAL, est destinée au dépôt et à la diffusion de documents scientifiques de niveau recherche, publiés ou non, émanant des établissements d'enseignement et de recherche français ou étrangers, des laboratoires publics ou privés. 
(c) 2013 IEEE. Personal use of this material is permitted. Permission from IEEE must be obtained for all other users, including reprinting/ republishing this material for advertising or promotional purposes, creating new collective works for resale or redistribution to servers or lists, or reuse of any copyrighted components of this work in other works.

\title{
Real-time Accelerometer Coupled Self-Mixing Laser Displacement Sensor for Embedded Applications
}

\author{
U. Zabit, O. D. Bernal, A. Chamorro-Coloma and T. Bosch \\ Univ de Toulouse, INP, LAAS, F-31400 Toulouse, France \\ CNRS, LAAS, 7 avenue du Colonel Roche, F-31400 Toulouse, France
}

\begin{abstract}
A Solid-State Accelerometer (SSA) coupled Self-Mixing (SM) interferometric laser displacement sensor is presented that is able to provide correct measurements in real-time while being subject to extraneous movements. Such a SSA-SM sensor is thus able to correct the influence of parasitic movements that would corrupt true target motion sensing. Hence, it can be used for those embedded applications where a sensor needs to operate under non-stationary conditions. The SSA-SM sensor achieves this robustness through a real-time data fusion between the SM interferometric signal and the acceleration signal. The falsified displacement signal retrieved by processing the SM interferometric signal (representing the sum of true target movement and the extraneous movement) is thus corrected in real-time. Our SSA-SM sensor has been compared with a reference commercial sensor and it has a precision of $96 \mathrm{~nm}$ rms with a bandwidth of $40 \mathrm{~Hz}-500 \mathrm{~Hz}$.
\end{abstract}

\section{INTRODUCTION}

Self-mixing (SM) or optical feedback interferometry technique has been widely used for metrological sensing applications during the last two decades as it results in a simple, compact, self-aligned, and low-cost sensor [1].

SM effect occurs in a laser when a part of the beam backscattered by a target is coupled back into the laser cavity and causes interference with the emitted beam, thus modifying the spectral properties of the laser. The variations in the optical output power of the laser diode $P(t)$ caused by this optical feedback can be written as [2]:

$$
P(t)=P_{0}\left[1+m \cdot \cos \left(x_{F}(t)\right)\right]
$$

where $P_{0}$ is the emitted optical power under free-running conditions, $m$ is the modulation index and $x_{F}(t)$ is the laser output phase in the presence of feedback, given by:

$$
x_{F}(t)=2 \pi \frac{D(t)}{\lambda_{F}(t) / 2}
$$

where $D(t)$ is the target displacement.

The emission wavelength subject to feedback $\lambda_{F}(t)$ is given by the phase equation [3]:

$$
x_{0}(t)-x_{F}(t)-C \sin \left[x_{F}(t)+\arctan (\alpha)\right]=0
$$

where $\alpha$ is the linewidth enhancement factor and $x_{0}(t)$ is the laser output phase in the absence of feedback, found by replacing $\lambda_{F}$ with $\lambda_{0}$ in eq. 2 , where $\lambda_{0}$ is the emission wavelength under free running conditions.

The feedback coupling factor $C$ is given by

$$
C=\frac{\tau_{D}}{\tau_{L}} \gamma \sqrt{1+\alpha^{2}} \kappa_{e x t}
$$

where $\tau_{L}$ and $\tau_{D}$ are the round trip times in the internal and external cavities respectively, $\gamma$ is the coupling efficiency and $\kappa_{e x t}$ depends linearly on the surface reflectivity of the target. The $C$ parameter plays a very important role in the SM signals as variations in $C$ cause changes in the so called SM operating regimes [1], where each regime would require a specific signal processing for the sake of displacement extraction [2]. For the present work, care has been taken to maintain the SM signal in the moderate regime with $C$ ranging from 1 to 4 due to the fact that such a regime requires simplified processing and is not affected by the fringe-loss issue [4].

It needs to be mentioned that SM displacement sensors have traditionally needed a stationary support (such as an optical table and/or anti-vibration material) to guarantee an accurate target measurement as any movement of the SM laser sensing system can corrupt the true target displacement measurement. Such a situation has thus restricted the use of SM sensors for embedded applications.

So, to overcome such a limitation, an accelerometer coupled SM sensor has been proposed [5] that measures true target displacement even if the laser head undergoes unwanted displacements (see Fig. 1). The solid-state MEMS (microelectro-mechanical system) accelerometer measures such parasitic displacements of the laser sensor and a subsequent subtraction of these parasitic displacements from the falsified SM displacement provides the correct target displacement. 
(c) 2013 IEEE. Personal use of this material is permitted. Permission from IEEE must be obtained for all other users, including reprinting/ republishing this material for advertising or promotional purposes, creating new collective works for resale or redistribution to servers or lists, or reuse of any copyrighted components of this work in other works.

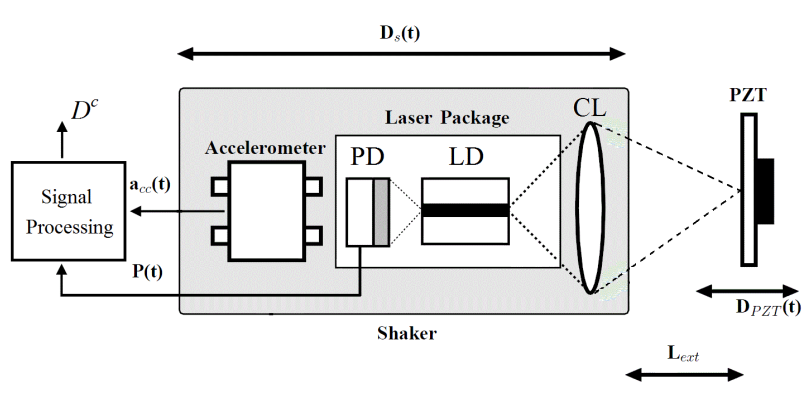

Figure 2. Block diagram of the Solid-State Accelerometer coupled Self-Mixing (SSA-SM) sensor: photodiode (PD), laser diode (LD), collimating lens (CL), and piezoelectric transducer (PZT).

Nonetheless, it needs to be said that the approach proposed in [5] was completely offline and the three main processing blocks i.e. 1) displacement retrieval from SM signal, 2) displacement retrieval from acceleration signal provided by a Solid-State Accelerometer (SSA), and 3) Phase and Gain matching were all processed separately on a PC.

The present paper, however, presents the results of a realtime data fusion between the SM signal provided by the LD and the acceleration signal $\left(\mathrm{a}_{\mathrm{cc}}\right)$ provided by the SSA (see Fig. 1 and Fig. 2). The SM signal has been processed using the real-time method of Consecutive-Samples-based-Unwrapping (CSU) that has been detailed in [6]. $\mathrm{a}_{\mathrm{cc}}$ has been filtered and integrated twice using analog circuits whereas the phase and gain matching (to be explained in the following sections) has been done using a Digital Signal Processor (DSP).

The following sections describe the signal processing and experimental set-up that allowed achieving a real-time data fusion between the SM signal and the $\mathrm{a}_{\mathrm{cc}}$ signal so that a laser displacement sensor can be used for those embedded applications where the presence of extraneous/parasitic movements would normally falsify the sensor measurement.

\section{SignAL PROCESSING}

Fig. 2 details the three main steps undertaken for the retrieval of true target displacement even in presence of parasitic displacements affecting the SM sensor.

\section{A. Optical self-mixing signal}

The variations in the optical output power of the laser diode $\mathrm{P}(\mathrm{t})$ are monitored through the built-in photodiode contained in the laser diode package. The SM based displacement is retrieved by extracting the phase from $\mathrm{P}(\mathrm{t})$ by using the already-mentioned CSU method [6] that allows displacement measurements with a precision of roughly $\lambda / 8$.

This displacement based on the optical signal is denoted here as $D_{\Sigma}$ as it is a sum of the true target displacement $D_{\text {PZT }}$ and the parasitic displacement $D_{s}$.

The CSU processing starts by the detection of self-mixing interferometric fringes, where each fringe represents a $\lambda / 2$ movement. This initial step is quite similar to the first step of

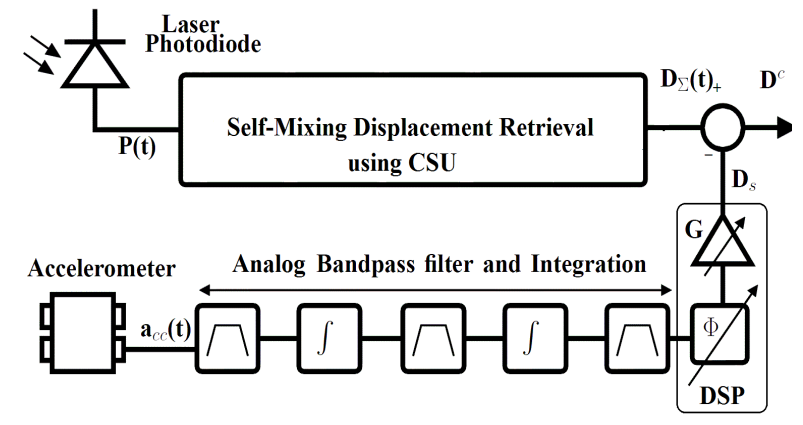

Figure 1. Block diagram of the signal processing required for the real-time SSA-SM sensor: CSU (Consecutive-Samples-basedUnwrapping) and DSP (Digital Signal Processor).

the PUM [3] or the fully analog SM processing method [7]. Then, instead of doing an iterative joint-estimation as in PUM, it recovers the displacement by adding the fringe-counting based staircase signal to the normalized SM signal, as it is also done in [7]. As compared to a complex approach such as the PUM, previously used in [5], that is based on joint-estimation of SM parameters requiring iterative optimization routines, the CSU method has the benefit of requiring only two SM samples to update the corresponding displacement output. This then allows a fast real-time displacement measurement at a price of a relatively lower measurement precision [6].

An integrated micro-converter AduC7020 from Analog Devices ${ }^{\circledR}$ working at 40 MIPS has been used to implement CSU. It has integrated Analog-to-Digital (A/D) and Digital-toAnalog (D/A) converters. The incoming signal $\mathrm{P}(\mathrm{t})$ is processed into the output displacement signal $D_{\Sigma}(t)$ at $125 \mathrm{KHz}$. So, the output displacement is updated every $8 \mu \mathrm{s}$.

\section{B. Acceleration signal}

As already mentioned, $\mathrm{D}_{\mathrm{s}}$ is recovered by using a solidstate accelerometer. In order to do so, the acceleration signal $\mathrm{a}_{\mathrm{cc}}(\mathrm{t})$ is filtered and integrated twice, as indicated in the lower half of Fig. 2. The filtering is done over the operating bandwidth of $40 \mathrm{~Hz}-500 \mathrm{~Hz}$ so that low-frequency drifts that can disturb the subsequent integration steps as well as high frequency acceleration signal saturation can be effectively avoided.

The choice of the employed SSA has a direct consequence on the overall SSA-SM sensing system precision. It was thus shown in [8] that the precision of an accelerometer coupled SM sensor can be greatly improved by the use of a low noise accelerometer. Thus, a six-fold improvement in system resolution was achieved by using LIS344ALH instead of ADXL311 (that was initially used in [5]). It thus demonstrated that the overall system resolution can be limited by the noise resolution of the employed accelerometer.

Furthermore, it was shown in [5] that the displacement resolution of the SSA signal depends on the low cutoff frequency of the system. For a $20 \mathrm{~Hz}$ lower cutoff, LIS344ALH is thus expected to introduce an RMS error of approximately 40nm [8]. 
(c) 2013 IEEE. Personal use of this material is permitted. Permission from IEEE must be obtained for all other users, including reprinting/ republishing this material for advertising or promotional purposes, creating new collective works for resale or redistribution to servers or lists, or reuse of any copyrighted components of this work in other works.

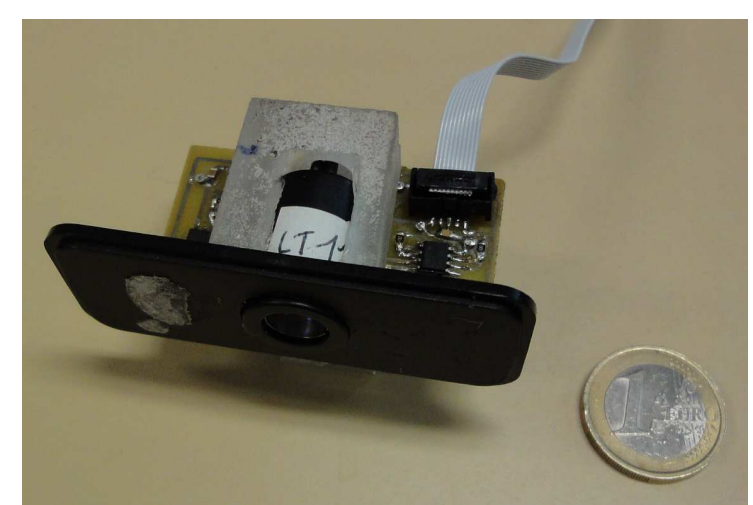

Figure 3. The Solid-State Accelerometer coupled Self-Mixing (SSASM) sensor head that can be deployed for embedded applications. Note that signal processing units are not shown in this picture.

For the present real-time data fusion, LIS344ALH has been used. The integration and filtering stages (indicated in Fig. 2) needed for extracting displacement from LIS344ALH have been based on standard analog OP-AMP based circuits.

\section{Phase and Gain Adjustments}

Finally, in order to match the two signals $\left(D_{\Sigma}\right.$ and $\left.D_{s}\right)$, phase $(\phi)$ and gain $(\mathrm{G})$ adjustments are done. This is necessary as both signals i.e. the SM signal and the acceleration signal pass through their respective signal amplification and acquisition circuits that introduce differences in phase and gain between the two signals. This signal matching of the two signals is followed by the last step of their subtraction that allows us to correctly recover the true target displacement $\mathrm{D}^{\mathrm{c}}$.

An integrated DSP from Microchip ${ }^{\circledR}$, called the dsPIC33FJ128GP has been used for the present real-time data fusion. It has also got integrated $\mathrm{A} / \mathrm{D}$ and $\mathrm{D} / \mathrm{A}$ converters that allow a simple interfacing. The incoming double integrated signal is processed into the gain and phase adjusted displacement signal $D_{s}(t)$ at $10 \mathrm{KHz}$ (see Fig. 2). Such a sampling rate is sufficiently high to correctly process the signal belonging to $40 \mathrm{~Hz}-500 \mathrm{~Hz}$ operating bandwidth.

In order to measure the phase and gain coefficients between the two displacements signals, the SSA-SM sensor was subjected to vibrations within the $40 \mathrm{~Hz}-500 \mathrm{~Hz}$ bandwidth. This allowed us to recover the coefficients that have been presented in Fig. 4.

Then, a custom filter was designed based on these coefficients and it was also implemented on our DSP. Such a filter thus allowed us to match $D_{\Sigma}$ with $D_{s}$ (see Fig. 2).

Finally, after this phase and gain matching of the two signals $\left(D_{\Sigma}\right.$ and $\left.D_{s}\right)$, we need only perform the last step of their subtraction that allows us to obtain the corrected displacement $\mathrm{D}^{\mathrm{c}}$. Such a solution thus allows us to recover corrected displacement in real-time.

\section{EXPERIMENTAL SET-UP}

The experimental set-up used to validate the working of our real-time accelerometer coupled SM laser displacement
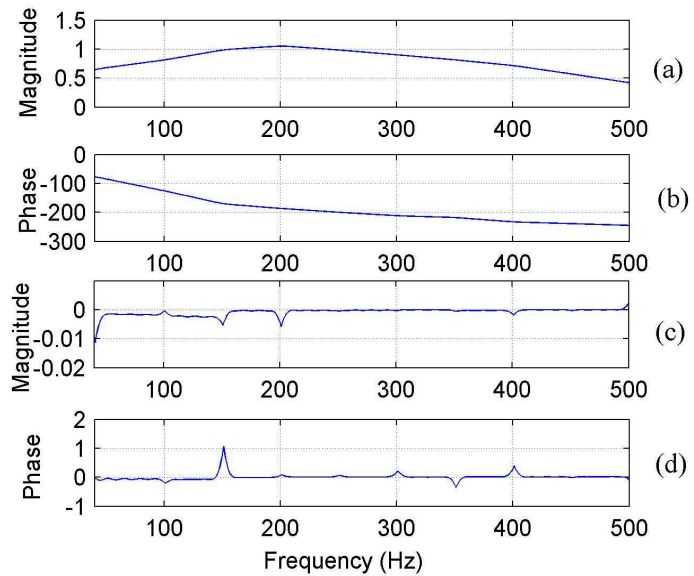

Figure 4. DSP filter Bode diagram: (a) gain transfer function, (b) phase transfer function in degrees. (c) and (d) gain and phase error in degrees between the desired and the implemented filter.

sensor is shown in Fig. 1. As already mentioned, the used SSA is LIS344ALH accelerometer from $\mathrm{ST}^{\circledR}$ (typical noise resolution of $50 \mu \mathrm{g} / \mathrm{Hz}^{1 / 2}$ and a full-scale range of $\pm 2 \mathrm{~g}$ ). The SM sensor is based on a Sanyo ${ }^{\circledR}$ DL7140 laser diode emitting at $\lambda=785 \mathrm{~nm}$ with an output power of $50 \mathrm{~mW}$. The SSA was glued on the SM sensor laser head so that it could measure as faithfully as possible the parasitic movement of the SM sensor head (see Fig.3).

The SSA-SM sensor head was mounted on a mechanical shaker (Fig. 1) that was used to generate vibrations undergone by the sensor. We used a commercial PZT (piezoelectric transducer) actuator from Physik Instrumente (P753.2CD) as target. This device has a built-in capacitive feedback sensor with $2 \mathrm{~nm}$ resolution that was subsequently used as a reference sensor for the PZT movement.

\section{EXPERIMENTAL RESULTS}

Let us now look into some of the experimental results for the SSA-SM sensing system.

Initially, in order to validate if the SSA-SM sensing system was able to eliminate the influence of extraneous parasitic movements, it was decided to keep the target stationary while only the shaker was excited. It was thus measured that the real-time SSA-SM sensing system eliminated such a parasitic vibration to an RMS value of $97 \%$ or a reduction of $31 \mathrm{~dB}$ of the extraneous parasitic motion. The measurement precision was found to be approximately $96 \mathrm{~nm}$.

Subsequently, after this validation, different tests were conducted to verify a correct working of our real-time SSASM system for the sake of target displacement measurements. For this purpose, the shaker and the PZT were both excited with either harmonic or arbitrary excitation signals.

Fig. 5 presents the correction result for the case where the target i.e. the PZT was made to vibrate at $82 \mathrm{~Hz}$ with peak to peak amplitude of $5 \mu \mathrm{m}$ while the extraneous harmonic 
(c) 2013 IEEE. Personal use of this material is permitted. Permission from IEEE must be obtained for all other users, including reprinting/ republishing this material for advertising or promotional purposes, creating new collective works for resale or redistribution to servers or lists, or reuse of any copyrighted components of this work in other works.

movement acting on the SSA-SM sensor head (provided by the shaker) was at $225 \mathrm{~Hz}$. It can be seen in Fig. 5 (purple

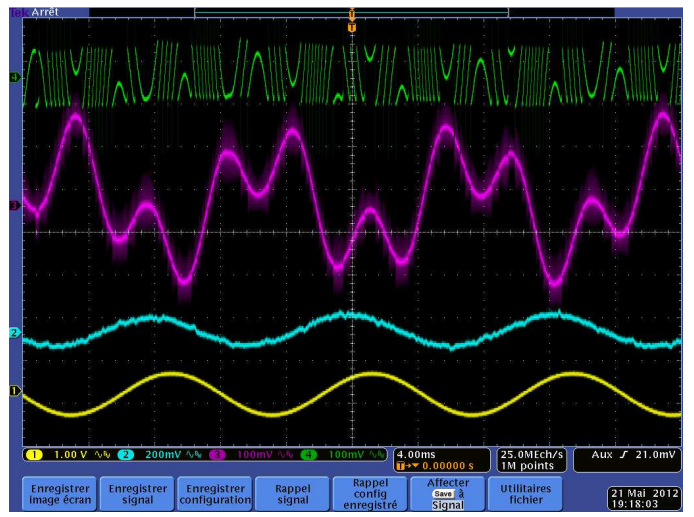

Figure 5. Experimental real-time sinusoidal vibration correction for an extraneous sinusoidal movement at $225 \mathrm{~Hz}$ while the target vibrates at $82 \mathrm{~Hz}$ : (green) SM signal, (purple) corrupted movement, (blue) corrected movement, and (yellow) reference sensor movement.

curve) that the corrupted signal i.e. $\mathrm{D}_{\Sigma}$ is not at all indicative of the true target movement (seen in Fig. 5 yellow curve). However, a correct target displacement measurement has been made by our real-time SSA-SM sensing system as indicated by the blue curve of Fig. 5. A close resemblance between the corrected signal and the reference signal is thus seen.

Another real-time displacement measurement result is presented in Fig. 6 where the target i.e. the PZT was excited by an arbitrarily shaped excitation signal composed of $20 \mathrm{~Hz}-$ $40 \mathrm{~Hz}-100 \mathrm{~Hz}-140 \mathrm{~Hz}$ while the shaker was excited at $50 \mathrm{~Hz}$. As a consequence, the displacement $\mathrm{D}_{\Sigma}$ (Fig. 6 purple curve) retrieved from the SM signal (Fig. 6 green curve) is corrupted and is not entirely indicative of true target movement (Fig. 6 yellow curve). However, the influence of the extraneous movement disturbing the SSA-SM sensing system has been correctly eliminated by our real-time signal processing which allows a correct arbitrary target movement measurement as seen in Fig. 6 blue curve.

\section{CONCLUSION}

In this paper, a real-time data fusion between a SelfMixing (SM) interferometric laser diode (LD) based displacement sensor and a Solid-State Accelerometer (SSA) has been presented. The said real-time SM displacement sensor system is based on a LD package from Sanyo ${ }^{\circledR}$ (DL7140) emitting at $\lambda=785 \mathrm{~nm}$. The accelerometer LIS344ALH is from $\mathrm{ST}^{\circledR}$ with a typical noise resolution of $50 \mu \mathrm{g} / \sqrt{ } \mathrm{Hz}$. Real-time signal processing has been achieved by an embedded micro-converter, a DSP, and analog OP-AMP based integrator and filter circuits.

The presented real-time SSA-SM sensor allows measuring correct target movements even when the LD based SM sensor is subject to extraneous movements. This results in a displacement or vibration sensing system that can be used for embedded applications as there is no more need of keeping the sensor stationary. Such an approach opens the way for the use

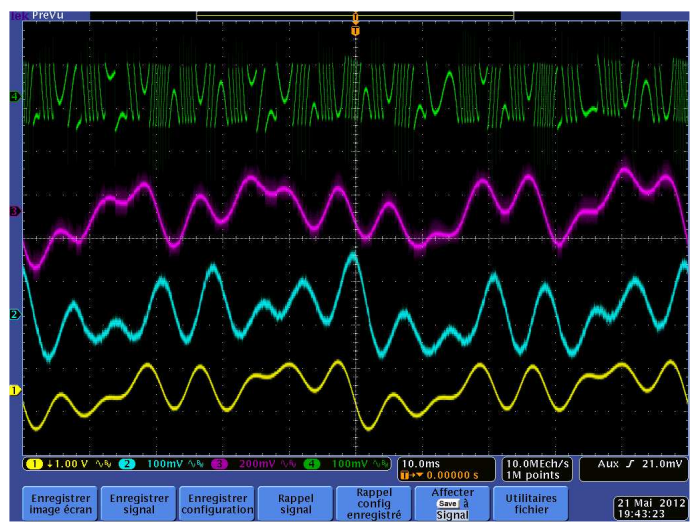

Figure 6. Experimental real-time correction of an arbitrary target movement corrupted by a different arbitrary movement acting on the SSA-SM sensor: (green) SM signal, (purple) corrupted movement, (blue) corrected movement, and (yellow) reference sensor movement.

of such laser sensors in conditions where the use of antivibration support is not available or possible. The presented real-time data fusion between the acceleration signal and the SM interferometric signal thus results in a robust, compact and low-cost sensing system.

\section{ACKNOWLEDGEMENT}

This research work has been funded in part by the Agence Nationale de la Recherche (ANR), France under the contract ANR-2011-EMMA-001-01.

\section{REFERENCES}

[1] S. Donati, "Developing self-mixing interferometry for instrumentation and measurements”, Laser \& Photon. Rev., 6: 393417, May 2012.

[2] U. Zabit, T. Bosch, and F. Bony "Adaptive transition detection algorithm for a self-mixing displacement sensor", IEEE Sensors Journal, vol. 9, Issue 12, pp. 1879-1886, 2009.

[3] C. Bes, G. Plantier, and T. Bosch, "Displacement measurements using a self-mixing laser diode under moderate feedback", IEEE Trans. Instr. \& Measur., vol. 55, no. 4, pp. 1101-1105, 2006.

[4] U. Zabit, T. Bosch, F. Bony and A. D. Rakic, "A self-mixing displacement sensor with fringe-loss compensation for harmonic vibrations", IEEE Photonics Technology Letters, vol. 22, No. 6, pp. 410-412, 2010

[5] U. Zabit, O. D. Bernal, T. Bosch, and F. Bony, "MEMS accelerometer embedded in a self-mixing displacement sensor for parasitic vibration compensation," Optics Letters, vol. 36, pp. 612-614, 2011.

[6] U. Zabit, O. D. Bernal, and T. Bosch, Zabit, "Self-mixing sensor for real-time measurement of harmonic and arbitrary displacements", Instrumentation and Measurement Technology Conference (I2MTC), 2012 IEEE International, vol., no., pp.754-758, 13-16 May 2012.

[7] M. Norgia, and A. Pesatori, "Fully analog self-mixing laser vibrometer", Instrumentation and Measurement Technology Conference (I2MTC), 2011 IEEE, vol., no., pp.1-4, 10-12 May 2011.

[8] U. Zabit, O. D. Bernal, and T. Bosch, "A self-mixing displacement sensor compensating parasitic vibration with a MEMs accelerometer", Sensors, 2011 IEEE , vol., no., pp.1386-1389, 28-31 Oct. 2011. 